\title{
Laboratorio Ludo-Matemático, Herramienta Potenciadora del Pensamiento Geométrico-Métrico en Preescolar ${ }^{1}$
}

\section{Ludo-Mathematical Laboratory, Geometric-Metric Thinking Potentiator in Preschool}

\author{
DOI: http://dx.doi.org/10.17981/cultedusoc.9.3.2018.62 \\ Artículo de investigación. Fecha de recepción: 15/06/2018. Fecha de aceptación: 27/11/2018 \\ Ana Pérez-Pisciotti²; \\ Miladys Torres-Ospino; Maura Torres-Rojas; Nancy Rojas-De Sánchez; \\ Neris Bastidas-Miranda; Amín Vilardy-Torres; Daniris Rivas-Alvarado; \\ Leonardo Martínez-Chimenty; Martha Ríos-Colorados; Mireya Rojas-Martínez; \\ Oriana Rangel-Campo; Raquel Cadena-Caro y Yohana Oliveros Oliveros ${ }^{3}$ \\ IED Roberto Robles de Algarrobal, sede educativa EMR \\ Camilo Torres-Col. Dptal. Bto. Carlos Vilardy (Colombia) \\ andrejosue2016@gmail.com
}

Para citar este artículo:

Pérez-Pisciotti, A., Torres-Ospino, M., Torres-Rojas, M., Rojas-De Sánchez, N., Bastidas-Miranda, N., Vilardy-Torres, A., Rivas-Alvarado, D., Martínez-Chimenty, L., Ríos-Colorados, M., Rojas-Martínez, M., Rangel-Campo, O., Cadena-Caro, R. y Oliveros, Y. (2018). Laboratorio Ludo-Matemático, Herramienta Potenciadora del Pensamiento Geométrico-Métrico en Preescolar. Cultura. Educación y Sociedad 9(3), 521-528. DOI: http://dx.doi.org/10.17981/cultedusoc.9.3.2018.62

\section{Resumen}

El aprendizaje de las matemáticas al inicio de la vida escolar, resulta un reto para el educador, por la complejidad que los contenidos tienen, razón. El objetivo del presente estudio fue identificar los conocimientos que los estudiantes tienen sobre las matemáticas para crear un laboratorio ludo-matemático sustentado en el uso de tics como herramienta potenciadora del pensamiento geométrico-métrico en preescolar. La investigación se sustenta desde la mirada cualitativa, y se trabajó con los maestros de la sede Camilo Torres de algarrobal, del Municipio del Banco Magdalena. Como instrumento de recolección de la información se realizó una entrevista estructurada. Los resultados permiten mostrar que los estudiantes en sus primeros años escolares se les dificultan el aprendizaje de las matemáticas, sin embargo, tiene conocimientos básicos. En cuanto a la motivación y participación se encuentra que le es de mayor motivación aprender si los espacios son interactivos y recreativos.

Palabras clave: Laboratorio de Matemáticas; Pensamiento Geométrico Métrico; Lúdica; saber; escuela.

\section{Abstract}

The learning of mathematics at the beginning of school life, is a challenge for the educator, because of the complexity that the contents have, reason. The objective of the present study was to identify the knowledge that students have about mathematics to create a ludo-mathematical laboratory based on the use of tics as an enhancing tool of geometrical-metric thinking in preschool. The research is based on a quantitative view, and the teachers of the Camilo Torres de Algarrobal headquarters in the Municipality of Banco Magdalena were worked on. As an instrument for gathering information, a structured interview was conducted. The results allow to show that the students in their first school years are difficult to learn mathematics, however they have basic knowledge. Regarding motivation and participation, we find that it is more motivating to learn if the spaces are interactive and recreational.

Keywords: Mathematics Laboratory; Metric Geometric Thought; Playful to know; school.

\footnotetext{
1 Este artículo ha sido derivado del Programa de Fortalecimiento de la Cultura Ciudadana y Democrática CT+I a través de la IEP apoyada en TIC en el Departamento de Magdalena: CICLON.
}

2 Líder del grupo de investigación "Medidores del mundo".

3 Docentes de la IED Roberto Robles de Algarrobal, sede educativa EMR Camilo Torres-Col. Dptal. Bto. Carlos Vilardy. Grupo de Investigación "Medidores del Mundo". 2017 Magdalena-Colombia. Universidad de la Costa CUC.

- The author; licensee Universidad de la Costa - CUC. 


\section{Introducción}

La comprensión y uso de las matemáticas beneficia el desarrollo del lenguaje pues exige la interpretación y codificación de información, su organización y su uso para obtener nuevas conclusiones; tanto la interpretación como la comunicación de la información se hace mediante la expresión gráfica, oral y escrita. Asimismo, no se puede desconocer la utilidad propia que tienen las matemáticas tanto en el mundo científico como en el desarrollo tecnológico.

El objetivo entonces, de la presente investigación es identificar los conocimientos que los estudiantes tienen sobre las matemáticas para crear un laboratorio ludo-matemático sustentado en el uso de tics como herramienta potenciadora del pensamiento geométrico-métrico en preescolar de la sede Camilo Torres de Algarrobal, del Municipio del Banco Magdalena.

La educación es objeto, es ambiente en el que el sujeto se piensa, se define, se reconoce bajo un componente cultural propio, miembro de una familia particular y en un medio social específico. Es allí donde cada individuo debe encontrar su realización como un ser en armonía.

"No existe la educación, sino porque hay un sujeto educable que le da sentido y además el sistema educativo debe preparar para esto, su misión es prepararlos para el mundo. Vivimos de la muerte de nuestras células, así como una sociedad vive de la muerte de sus individuos, lo que le permite rejuvenecer" (Pino, 2013).

El medio está involucrado en un difícil proceso de cambio. Una transformación que no es organizada y planificada afecta el trabajo, aprendizaje y resultados. El aprendizaje de las matemáticas, es un tema que siempre debe de ser abordado, y que se potencializan en la edad escolar, en un medio de realización humana más que en un fin como ciencia que se debe ser aprendida, y debe brindarle al individuo la posibilidad de superar retos y obstáculos, de proponerse alternativas de solución, de analizar y obrar por un cambio (Gonzales, 2010).

"Esa realización noes solamenteaxiológica; es un desarrollo humano que tiene un compromiso fundamental en el desarrollo lógico, definitivo como estrategia para conocer el mundo, apropiar y cambiar su cultura, abarcar una situación nueva con la posibilidad de desglosarla (análisis), organizar una información de acuerdo con unas prioridades (clasificación y seriación), proponer alternativas de acción (transferencia y síntesis), tomar decisiones y así lograr la autonomía intelectual, fundamento de la autonomía moral" (Cifuentes, 2013).

"El conocimiento en el componente geométrico-métrico es fundamental para todo ser humano, puesto que lo aplica en la vida cotidiana; por ejemplo, estamos rodeados de diferentes objetos y formas geométricas los que podemos estudiar de forma experimental sus lados, sus bordes y aplicar los conocimientos adquiridos, al observar un árbol ahí existe la geometría, ya que si requiere cortarlo deben conocer a qué distancia se encuentra de una edificación y saber con precisión el ángulo al que lo puede dirigir al derribarlo no cause daños, se pueden ver ángulos y otras formar. Una edificación es geometría, todo al alrededor necesita obligatoriamente conocimientos geométricos; para medir un terreno, hacer un huerta, saber cuántos metros cuadrados puede trazar, cuántas plantas puede sembrar y ayudarle a los padres a su quehacer diario, en su finca asimismo se encuentran actividades de la vida diaria relacionadas con las compras en el supermercado, con la cocina, con los deportes, con la lectura de mapas, con la construcción, etc., acercan a los estudiantes a la medición y les permiten desarrollar muchos conceptos y destrezas matemáticas" (Londoño 2010). 
Marín, (2013)

"El uso de actividad lúdica atiende necesidades de estrategias que permite la enseñanza de la Geometría de una forma práctica para los niños, además es indispensable en la primera etapa escolar ya que, si se utiliza la lúdica, adquiere aprendizaje significativo en el niño, permitiéndole participar" (2013).

Es importante destacar que las actividades lúdicas despiertan en el niño una atracción hacia la materia.

"El niño muestra un interés especial si al enseñarle las matemáticas se utilizan a través de actividades lúdicas, Propiciando en cada niño la oportunidad de moldear libremente su vida y participar del constante cambio con la aparición de las nuevas tecnologías en el ámbito educativo" (Pérez, 2011).

Arboleda (2011), argumenta que se debe contemplar tres aspectos importantes en el proceso educativo: a la apropiación social del conocimiento, Fortalecimiento de la comunidad científica, Generación de nuevo conocimiento. En donde se concluye lo importante de obtener que el estudiante aprenda a adquirir nuevas estrategias que le permitan sacar más provecho de su trabajo. Para ellos es necesario que realice actividades, compruebe los errores y se descubra la forma de evitarlos. La estrategia didáctica debe ser muy llamativa, ya que esta debe estimular la participación del estudiante para que sea este quien construya su propio aprendizaje, fomentando así la motivación y el interés en esta área.

Por otra parte (Núñez, 2010) sustenta que parte del gran interés y motivación que se despierta en los niños en el proceso educacional se deriva de su interacción con actividades significativas, donde se observa un mayor desarrollo en cuanto al pensamiento espacial y sistema geométrico, dichas actividades se deben ajustar a la edad e intereses y habilidad de los niños, de igual forma como aspecto muy importante se resalta el interés y habilidad que muestran los niños en lo relacionado con las actividades en el PC.

Para Gómez (2014), la construcción de una plataforma Moodle, diseñada especialmente para la enseñanza de matemáticas en el grado quinto y una de las unidades está especialmente dedicada a la enseñanza de la geometría matemática; está es una herramienta que permite integrar la tecnología como una forma de mostrar la enseñanza de esta asignatura con las diversas posibilidades de nuestro tiempo y acercarlos de una forma más de acuerdo a sus gustos, ya que la geometría es una asignatura que la encuentran en su entorno con una simple observación.

Según Ramírez (2013), el laboratorio de matemáticas se convierte en una estrategia que mejora los procesos de enseñanza-aprendizaje. Nuestra experiencia muestra como principales resultados docentes motivados, pensantes, creativos y cualificados; de igual manera, estudiantes que se convierten en mejores Seres Humanos, apropiados de conocimientos que le son útiles para sus vidas; haciendo aportes significativos a la sociedad, en tanto que formulan, plantean y resuelven problemas a partir de situaciones de la vida cotidiana, de las otras ciencias y de las matemáticas mismas.

Pero Hernández (2017), toma el aprendizaje como una palabra polisémica que es y ha sido definida desde diferentes teorías de la educación, sin embargo, cabe destacar que este im- 
plica cambio de actitudes y aptitudes cuando ha sido logrado; para el mismo influyen experiencias vividas dentro y fuera de la escuela, estrategias didácticas, actividades de enseñanza y aprendizaje, ambientes de aprendizaje, motivación, estilos de aprendizaje, estilos de enseñanza cuya finalidad es lograr que el alumno construya su propio aprendizaje en la vida y para la vida como un proceso permanente, flexible y dinámico.

Es así como la matemática implica el logro de ciertas competencias, así la competencia matemática integra el razonamiento lógico matemático a través de terminología, datos, destrezas, procedimientos, formulación y solución de problemas donde el alumno logre satisfacer, resolver y cubrir necesidades tanto personales como sociales (Hernández, 2017: Pérez-Acosta y Urrá, 2015).

Para Parrales y Tomalá (2016), la potencialización de la geometría actualmente es considerada como una de las bases fundamentales para evaluar el nivel de aprendizaje lógico matemático de los niños y adolescentes, este tipo de aprendizaje se caracteriza porque en los inicios de la vida escolar es aprendido mediante concreción con la realidad, no obstante debido a la complejidad que se presenta en el currículo, las habilidades cognitivas como: el análisis, la resolución de problemas, la comparación, el razonamiento, la demostración y la secuenciación de números. Proporcionar estrategias que permitan optimizar capacidades o habilidades de los estudiantes en lo que actualmente los docentes conocen, que la educación necesita cambios innovadores que manifiesten una actitud con predisposición al cambio.

\section{Laboratorio de Matemática}

Esta temática puede ser vista como una estrategia de enseñanza y aprendizaje; que le permite a los alumnos descubrir, relacionar, aplicar y construir su aprendizaje; porque en definitiva "Es necesario romper, con todos los medios, la idea preconcebida, y fuertemente atraída en nuestra sociedad, con probabilidad de obstáculos iniciales en la niñez de muchos, de que la matemática es necesariamente aburrida, abstrusa, inútil, inhumana y muy difícil" (Guzmán 2007, p.47 citado por Hernandez, 2017).

"Sobran razones para especificar que dentro de la asignatura de la matemática los ambientes de aprendizaje no deben quedar diseñados y relegados a la simple solución de ejercicios de forma mecánica y repetitiva una y otra vez, sino más bien deben permitir que el estudiante conozca y comprenda su entorno con ayuda de las matemáticas, porque realmente la finalidad no es aprender matemáticas por el simple hecho de hacerlo, sino más bien a decir" (Moreno, 2016), "Es aprender a través de las matemáticas... formas simbolizadas de nuestras propias experiencias... ideas importantes a través de una explicación simple" (Moreno, 2016), abrir los sentidos hacia lo que sucede desde nuestro interior hasta aquello que no logramos visualizar pero si lo podemos comprender.

Con base a lo anterior es fundamental generar propuestas que permitan la creación de ambientes favorecedores de aprendizaje de las matemáticas, siendo el laboratorio el que resultaría una buena alternativa, trascendiendo de lo magistral desde el docente, y también de la pasividad desde el estudiante, teniendo en cuenta el contexto y las formas de adquisición de conocimiento (Bosch, 2014). 
"El uso herramientas como lo son el pizarrón y marcadores es una parte de los materiales usados en el proceso de enseñanza y aprendizaje, sin embargo, ello no significa que sea lo único que se pueda hacer para crear un conjunto de actividades matemáticas para ser desarrolladas autónomamente por los participantes a través del uso de variados materiales, proceso que proporciona un ambiente de aprendizaje en el que se genera la relación entre actividad matemática y material manipulativo, relación que contribuye a la construcción y fundamentación de pensamiento matemático" (Arce s.f.p.2).

"El laboratorio de matemáticas en su formulación inicial ha buscado contribuir a la construcción del pensamiento matemático, favoreciendo así el acercamiento hacia una cultura matemática en el marco de un ambiente lúdico y recreativo. En tal sentido, puede considerarse como un dinamizador de construcción de pensamiento matemático a través del proceso de aprendizaje" (Pabón, Arce, Vega y Garzón 2011).

"La enseñanza de las matemáticas debe incluir los desarrollos de trabajo lúdicos, interactivos y experimentales en lo que estudiantes y profesores puedan intercambiar argumentos $\mathrm{y}$ desarrollar nuevas soluciones a antiguos problemas“ (UPN, 2014).

\section{Metodología}

La investigación estuvo orientada por el paradigma Crítico - Social, porque se fundamenta en la reflexión crítica, buscó la reforma de las instituciones que hacen parte de la realidad social a la que pertenecen los investigadores, La investigación fue de tipo cualitativo, como lo señala Cerda (2003), tuvo como objetivo la descripción de las cualidades de un fenómeno.
Técnicas e instrumentos de recolección de la información

Se empleó como instrumento la entrevista estructurada, redactada e impresa en físico, que consta de 3 preguntas y la observación directa, en donde se registraron acontecimientos y sucesos significativos dentro del proceso a través de registro de apuntes y fotográficos.

\section{Escenarios y actores del estudio}

El escenario en el que se desarrolló la investigación es la Institución Educativa Departamental IED Roberto Robles de Algarrobal. Sede Educativa EMR camilo con la participación de (25) docentes

\section{Procedimiento}

Momento (1): Se seleccionan los instrumentos a utilizar.

Momento (2): Se especifican las categorías de estudio.

Momento (3): Se escoge los instrumentos de recolección de información.

Momento (4): Con base a las categorías de estudio: Pensamiento matemático, herramientas de aprendizaje, tic; se aplicó a los participantes la entrevista, la cual pretende describir el fenómeno que se estudia partiendo del nivel de la investigación (no experimental) en donde se observa, y analiza tal y como acontece.

Momento (5): Una vez se tiene los resultados de la entrevista estructurada y la observación registrada se interpretan y comprenden los resultados a la luz de lo que exponen los teóricos que soportan la investigación.

Momento (6): Se relacionan los resultados y se muestran los hallazgos. 


\section{Resultados}

\begin{tabular}{cccc}
\hline $\begin{array}{c}\text { Categoría de } \\
\text { estudio }\end{array}$ & $\begin{array}{c}\text { Pregunta } \\
\text { orientadora }\end{array}$ & $\begin{array}{c}\text { Discurso de } \\
\text { los actores }\end{array}$ & $\begin{array}{c}\text { Articulación y } \\
\text { sistematización teórica }\end{array}$ \\
\hline
\end{tabular}

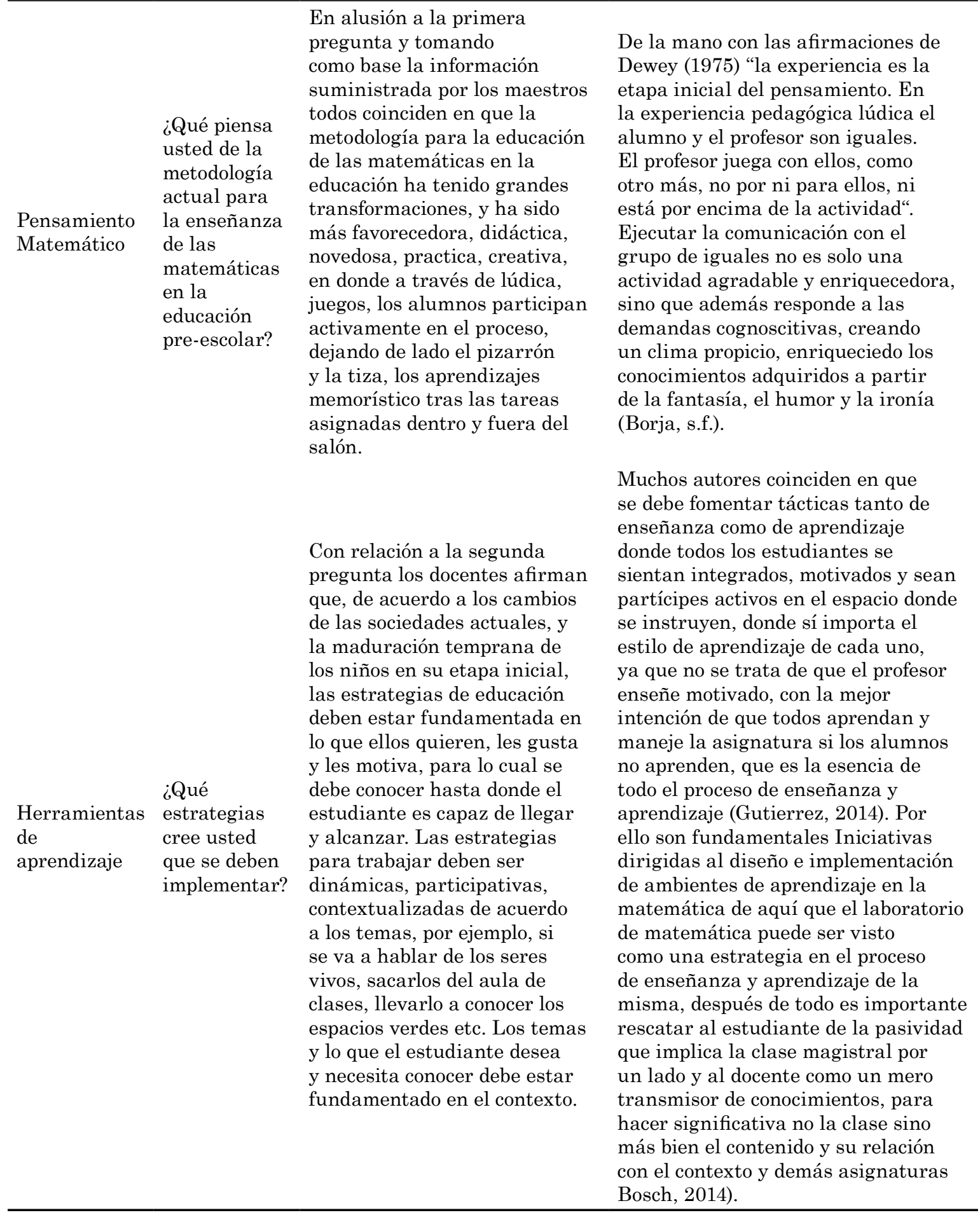


...

\begin{tabular}{|c|c|c|c|}
\hline $\begin{array}{l}\text { Categoría de } \\
\text { estudio }\end{array}$ & $\begin{array}{l}\text { Pregunta } \\
\text { orientadora }\end{array}$ & $\begin{array}{l}\text { Discurso de } \\
\text { los actores }\end{array}$ & $\begin{array}{l}\text { Articulación y } \\
\text { sistematización teórica }\end{array}$ \\
\hline TIC & $\begin{array}{l}\text { ¿Piensa que } \\
\text { las TIC son } \\
\text { un elemento } \\
\text { de apoyo para } \\
\text { el aprendizaje } \\
\text { de las } \\
\text { matemáticas? }\end{array}$ & $\begin{array}{l}\text { Finalmente, en la tercera } \\
\text { pregunta, todos los maestros } \\
\text { coinciden que la las TIC han } \\
\text { llegado a transformar los } \\
\text { entornos educativos, como } \\
\text { herramienta pedagógica } \\
\text { transforma los escenarios } \\
\text { de aprendiza, incentiva la } \\
\text { participación y motivación de } \\
\text { los estudiantes, crea ambientes } \\
\text { innovadores de enseñanza, } \\
\text { fomenta a la indagación desde } \\
\text { las edades tempranas, potencia } \\
\text { la practica conectando las } \\
\text { necesidades del estudiante con } \\
\text { la practica digital e interactiva. } \\
\text { Y es en el aprendizaje de las } \\
\text { matemáticas que se convierte } \\
\text { en una herramienta de gran } \\
\text { ayuda por los contenidos } \\
\text { propios de la asignatura. }\end{array}$ & $\begin{array}{l}\text { Las TIC se han convertido en un } \\
\text { elemento en la vida de los individuos, } \\
\text { y una herramienta estrategia que ha } \\
\text { favorecido a las nuevas dinámicas } \\
\text { del sistema educativo, siempre y } \\
\text { cuando sea desde el uso pedagógico. } \\
\text { En cuanto a la enseñanza de las } \\
\text { matemáticas permite incluir los } \\
\text { desarrollos de trabajo lúdicos, } \\
\text { interactivos y experimentales en } \\
\text { lo que estudiantes y profesores } \\
\text { puedan intercambiar argumentos } \\
\text { y desarrollar nuevas soluciones a } \\
\text { antiguos problemas (UPN, 2014). } \\
\text { Involucra la comprensión del espacio, } \\
\text { el desarrollo del pensamiento visual, } \\
\text { el análisis abstracto de figuras y } \\
\text { formas en el plano y en el espacio a } \\
\text { través de la observación de patrones } \\
\text { y regularidades, el razonamiento } \\
\text { geométrico y la solución de problemas } \\
\text { de medición, así como la construcción } \\
\text { de conceptos de cada magnitud } \\
\text { (Galvis, 2015). }\end{array}$ \\
\hline
\end{tabular}

Fuente: elaboración propia.

\section{Conclusiones}

Los docentes reconocen la importancia de ser participe activo en el desarrollo integral de sus educandos, por lo que se logra apropiar de estrategias, metodologías y lineamientos curriculares propios de la institución educativa, para hacer del laboratorio una experiencia significativa al proceso de enseñanza, logrando transmitir nuevos conocimientos a los demás grupos de trabajo.

Aunque son muchos los materiales lúdicos y didácticos y otros que se pueden optar para encaminar al discente al cambio de esa estructura magistral del aula e encaminarlo a un proceso experimental de construcción de conocimiento, no todo cumplen con los criterios estipulados coherentemente para llevar a cabo este tipo de metodología en la práctica educativa , y sobre todo para lograr los propósitos expuestos, debido al proceso lento con que se organiza el PEI, y procesos palpables que se tocaron en la descripción del problema del estudio en vía.

Finalmente, la consecución de un Laboratorio Ludo-Matemático es una de las estrategias que beneficiaria la formación estudiantil desde su fase inicial. El cuerpo de docente reconoce la importancia de atender a dicha debilidad que se presenta en los estudiantes y que se refleja en cada una de las áreas del conocimiento, y los beneficios que ofrece al proceso de enseñanza-aprendizaje, a la institución y a la contribución de un desarrollo integral. Afirman que componente geométrico-métrico va más allá de hacer figuras y tomar medidas, identifican la importancia y cómo repercute en cada competencia matemática y para la vida misma del educando. 


\section{Referencias}

Avendaño, I., Cortés, O. y Guerrero, H. (2015). Competencias sociales y tecnologías de la información y la comunicación como factores asociados al desempeño en estudiantes de básica primaria con experiencia de desplazamiento forzado $\mathrm{Di}$ versitas: Perspectivas en Psicología, 11(1). 13-36. Recuperado de http://www. redalyc.org/articulo.oa?id=67943296001

Camargo, L. (2011). El legado de Piaget a la didáctica de la Geometría. Revista Colombiana de Educación, 60.

Colorear, F. (2016). Figuras Geométricas -Dibujos para colorear. Recuperado de http://www.cicloescolar.com/2012/11/figuras-geometricas-dibujos-para.html [Accessed 16 Dec. 2016].

Godino, J (2012). Medida de magnitudes y su didáctica para maestros. Recuperado de https://www.ugr.es/ jgodino/

Guerrero, A. (2006). Desarrollo axiomático. Bogotá, D.C.: Editorial Ecoe.

Ludica.org. (2017). La Lúdica, el Constructivismo y el Aprendizaje Experiencial [online]. Recuperado de http://www.ludica.org/ Manual de convivencia, Institución Educativa La Rivera.

Herrera, B., Certain, R. y Calderón, M (2014). La norma hipotética fundamental desde el Paradigma Interpretativo. Justicia Juris, 10(2), 17-27. http://dx.doi. org/10.15665/rj.v10i2.324

Landinez-Lamadrid, D., Ramirez-Ríos, D., Neira, D., Parra, K. y Combita, J. (2017). Shapley Value: its algorithms and application to supply chains. INGE CUC, 13(1), 61-69. https://doi.org/10.17981/ingecuc.13.1.2017.06

Marcos, G. (2013). Un modelo de competencias matemáticas en un entorno interactivo. [Tesis doctoral]. Universidad de la Rioja, La Rioja, Recuperado de http:dialnet.unirioja.es/servlet/fichero_ tesis?.
Marín, D. (2014). Estrategias didácticas para fortalecer el pensamiento geométrico en estudiantes de grado sexto. [Tesis]. Universidad Católica de Manizales, Manizales.

OCDE. (2014). PISA. Marco de Evaluación y de Análisis de PISA para el Desarrollo. Lectura, Matemáticas y Ciencias. París: OCDE. Recuperado de https://www.oecd. org/pisa/aboutpisa/ebook\%20-\%20PISAD\%20Framework_PRELIMINARY\%20 version_SPANISH.pdf

Perfil, V. (2016). Cuerpos geométricos. Actividades. [online]. Recuperado de http:// aulasory.blogspot.com.co/2011/09/cuerpos-geometricos-actividades.html

Parrales, G. y Tomalá, B. (2016). La enseñanza de la geometría para la potencialización del aprendizaje lógico matemático en los estudiantes de séptimo grado de la escuela de educación básica Abdón Calderón Garaicoa, cantón La Libertad, provincia de Santa Elena, periodo lectivo 2015 - 2016. [Tesis]. Universidad Estatal Península de Santa Elena, La Libertad. Recuperado de http://repositorio.upse.edu.ec/handle/46000/3315

Pérez-Acosta, \& Urrá, M. (2015). El Premio Rubén Ardila a la investigación científica en psicología (2005-2015): De la psicología organizada a la historia de las ciencias del comportamiento. Cultura Educación y Sociedad, 6(2), 77-89. Recuperado de https://revistascientificas. cuc.edu.co/culturaeducacionysociedad/ article/view/1042/pdf_222

República de Colombia. Ministerio de Educación Nacional. (2011). Lineamientos curriculares. Recuperado de http://www. mineducacion.gov.co/lineamientos/matematicas/matematicas.pdf

Salamanca, M., Lombana, N. y Holguín, W. (2010). Uso de la robótica educativa como herramienta en los procesos de enseñanza. Ingeniería Investigación y Desarrollo, 10(1). 15-23. 